Respiration 2013;86:262-263

DOI: $10.1159 / 000348392$

\section{The Effect of Dithiothreitol on the Transcriptome of Induced Sputum Cells}

Torsten Goldmann ${ }^{a, c}$, Frauke Pedersen ${ }^{c, d}$, Sophie Seehase ${ }^{a, c}$, Sebastian Marwitz ${ }^{a, c}$, Dagmar S. Lang ${ }^{a, c}$, Anne-Marie Kirsten ${ }^{c, d}$, Peter Zabelb, , Ekkehard Vollmera, c, Helgo Magnussen ${ }^{c, d}$, Klaus F. Rabe ${ }^{c, d}$, Henrik Watz ${ }^{c, d}$

${ }^{\mathrm{a} C l i n i c a l}$ and Experimental Pathology and ${ }^{\mathrm{b}}$ Medical Clinic, Research Center Borstel and 'Airway Research Center North, Member of the German Center for Lung Research, Borstel, and dPulmonary Research Institute, LungenClinic Grosshansdorf, Grosshansdorf, Germany

Key Words

Induced sputum - Chronic obstructive pulmonary disease . Dithiothreitol · Transcriptome

\section{Findings}

Induced sputum is currently the best available noninvasive assessment of airway inflammation in chronic airway diseases such as asthma and chronic obstructive pulmonary disease (COPD). Accordingly, whole-genome gene expression profiling of induced sputum cells is increasingly being applied in the study of molecular disease mechanisms $[1,2]$. For the purpose of differential cell count analyses, sputum cell plugs are commonly lysed by dithiothreitol (DTT), which is used to reduce the disulfide bonds of mucus glycoproteins. However, DTT also directly affects other proteins in the sputum [3]. Thus, we assumed that DTT could also have an impact on the transcriptomes of induced sputum cells.

As part of a large-scale study that aims to explore molecular disease mechanisms in induced sputum by transcriptome analyses in COPD and asthma, we started to evaluate the effect of DTT on these transcriptomes. In order to address this issue, we applied a procedure recently described for bronchoalveolar lavage cells, which uses the HOPE technique for cell preservation with subsequent embedding in paraffin [4]. This technique allows preservation of nucleic acids and proteins for long-term storage of the samples [4].

The study was approved by the Ethics Committee of the Medical Association of Schleswig-Holstein, Bad Segeberg, Germany [reference number: AZ 030/11(II); all participants gave written informed consent]. We subjected RNA samples isolated from the sputum blocks (HOPE) of 4 COPD patients (all current smokers, $54.0 \pm 14.9$ pack years, mean age $63.5 \pm 9.5$ years, mean $\mathrm{FEV}_{1}$
$61.6 \pm 8.9 \%$ pred. and no respiratory tract infection/exacerbation or anti-inflammatory treatment within the 4 weeks prior to sputum induction). The samples were processed - with or without DTT - to transcriptome analysis, using an Agilent $(4 \times 44 \mathrm{~K})$ platform [4]. In order to obtain an overview of the general effects of DTT, pooled samples (600 ng each) of four RNA preparations from these patients - processed either with or without DTT - were analyzed. Processing of the sputum samples without DTT was performed by adding 8 volumes of PBS with subsequent mechanical homogenization of the sputum sample according to the method of Hector et al. [5]. Processing of the sputum samples with DTT followed a standard protocol adding 4 volumes of DTT to the sputum cell pellet.

The results are displayed in figure 1 . The comprehensive dataset is given in the online repository. We observed a strong effect of DTT on the transcriptomes with 2,906 genes differentially regulated on a logarithm base 2 value of the expression ratio (log-2 fold change) of higher than 2.0 (1,755 genes upregulated and 1,151 genes downregulated by DTT). On a log-2 fold change higher than 2.5, a total of 1,384 genes were still affected (917 genes upregulated and 467 downregulated by DTT). Among these, some play a role in COPD, such as interleukin 8 which was clearly upregulated by DTT (log-2 fold change $=5.2$ ). For a complete listing of all genes, see online supplementary material (www.karger.com/doi/10.1159/000348392). We conclude that the effect of DTT needs to be taken into account when transcriptome analysis is applied to induced sputum samples.

\section{Acknowledgements}

We thank Jasmin Tiebach, Maria Lammers, Petra Timm and Kirsten Paasch for excellent technical assistance.

\section{References}

1 Baines KJ, Simpson JL, Wood LG, et al: Transcriptional phenotypes of asthma defined by gene expression profiling of induced sputum samples. J Allergy Clin Immunol 2011;127:153-160.

2 Singh D, Fox SM, Tal-Singer R, et al: Induced sputum genes associated with spirometric and radiological disease severity in COPD ex-smokers. Thorax 2011;66:489-495.

3 Bafadhel M, McCormick M, Saha S, et al: Profiling of sputum inflammatory mediators in asthma and chronic obstructive pulmonary disease. Respiration 2012;83:36-44.

4 Marwitz S, Abdullah M, Vock C, et al: HOPE-BAL: improved molecular diagnostics by application of a novel technique for fixation and paraffin embedding. J Histochem Cytochem 2011;59:601-614.

5 Hector A, Jonas F, Kappler M, et al: Novel method to process cystic fibrosis sputum for determination of oxidative state. Respiration 2010;80: 393-400.

\section{KARGER}

E-Mail karger@karger.com

www.karger.com/res
2013 S. Karger AG, Basel

$0025-7931 / 13 / 0863-0262 \$ 38.00 / 0$ 


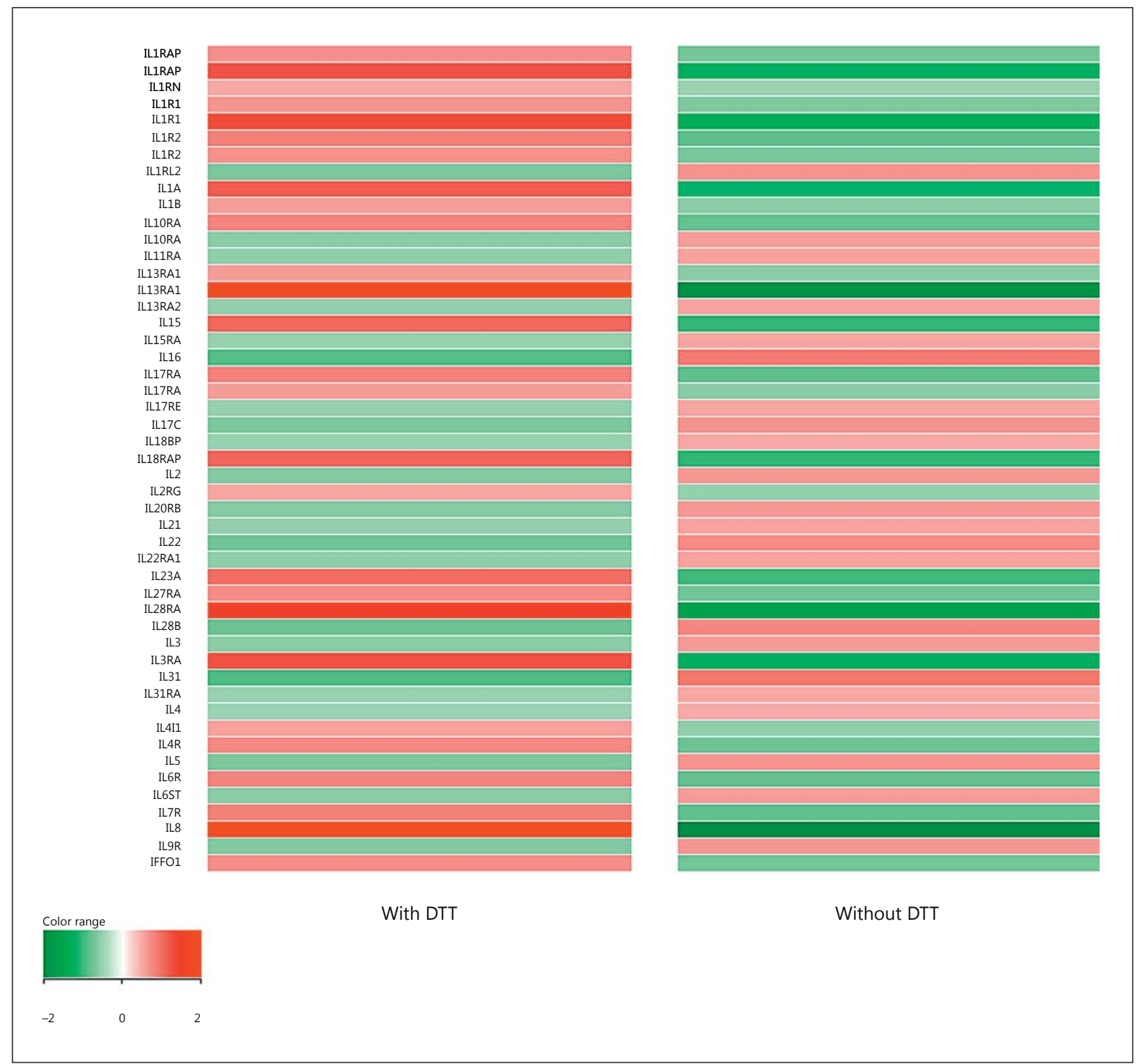

Fig. 1. Heat map of transcriptome analysis of HOPE-preserved induced-sputum RNA processed with and without DTT. Agilent (4 $\times 44 \mathrm{~K}$ ) human whole genome arrays were used. Analysis of the results was performed with a fold-change analysis using Gene-
Spring 12 software. Red color intensity indicates higher expression, green color intensity indicates lower expression and white indicates a medium level of expression of a gene in the comparison of both groups. Gene description is shown on the left-hand side. 